\title{
Tracing isolates from domestic human Campylobacter jejuni infections to chicken slaughter batches and swimming water using whole-genome multilocus sequence typing
}

\section{Kovanen, Sara}

2016-06-02

Kovanen , S , Kivisto , R , Llarena , A-K , Zhang , J , Karkkainen, U-M , Tuuminen , T , Uksila , J , Hakkinen , M , Rossi , M \& Hanninen, M-L 2016 , ' Tracing isolates from domestic human Campylobacter jejuni infections to chicken slaughter batches and swimming water using whole-genome multilocus sequence typing ' , International Journal of Food Microbiology , vol. 226 , pp. 53-60 . https://doi.org/10.1016/j.ijfoodmicro.2016.03.009

http://hdl.handle.net/10138/223995

https://doi.org/10.1016/j.ijfoodmicro.2016.03.009

publishedVersion

Downloaded from Helda, University of Helsinki institutional repository.

This is an electronic reprint of the original article.

This reprint may differ from the original in pagination and typographic detail.

Please cite the original version. 


\title{
Tracing isolates from domestic human Campylobacter jejuni infections to chicken slaughter batches and swimming water using whole-genome multilocus sequence typing
}

\author{
Sara Kovanen a , Rauni Kivistö a , Ann-Katrin Llarena a , Ji Zhang a , Ulla-Maija Kärkkäinen ${ }^{\text {b }}$, Tamara Tuuminen ${ }^{\text {c }}$, \\ Jaakko Uksila ${ }^{d}$, Marjaana Hakkinen ${ }^{\mathrm{e}}$, Mirko Rossi ${ }^{\mathrm{a}}$, Marja-Liisa Hänninen ${ }^{\mathrm{a}, *}$ \\ a Department of Food Hygiene and Environmental Health, University of Helsinki, Helsinki, Finland \\ ${ }^{\mathrm{b}}$ Eastern Finland Laboratory Centre Joint Authority Enterprise (ISLAB), Kuopio District Laboratory, Finland \\ c Eastern Finland Laboratory Centre Joint Authority Enterprise (ISLAB), Mikkeli District Laboratory and University of Helsinki, Department of Bacteriology and Immunology, Helsinki, Finland \\ d Keslab Laboratory, Department of Clinical Microbiology, Central Finland Health Care District, Jyväskylä, Finland and Fimlab Laboratories, Ltd., Jyväskylä, Finland \\ e Food and Feed Microbiology Research Unit, Research and Laboratory Department, Finnish Food Safety Authority, Evira, Finland
}

\section{A R T I C L E I N F O}

\section{Article history:}

Received 30 June 2015

Received in revised form 23 November 2015

Accepted 12 March 2016

Available online 20 March 2016

\section{Keywords:}

Domestically acquired campylobacteriosis

Summer peak

Chicken

Surface water

Source

\begin{abstract}
A B S T R A C T
Campylobacter jejuni is the leading cause of bacterial gastroenteritis and chicken is considered a major reservoir and source of human campylobacteriosis. In this study, we investigated temporally related Finnish human ( $\mathrm{n}=$ $95)$, chicken $(n=83)$ and swimming water $(n=20)$ C. jejuni isolates collected during the seasonal peak in 2012 using multilocus sequence typing (MLST) and whole-genome MLST (wgMLST). Our objective was to trace domestic human $C$. jejuni infections to $C$. jejuni isolates from chicken slaughter batches and swimming water. At MLST level, 79\% of the sequence types (STs) of the human isolates overlapped with chicken STs suggesting chicken as an important reservoir. Four STs, the ST-45, ST-230, ST-267 and ST-677, covered 75\% of the human and 64\% of the chicken isolates. In addition, 50\% of the swimming water isolates comprised ST-45, ST-230 and ST-677. Further wgMLST analysis of the isolates within STs, accounting their temporal relationship, revealed that 22 of the human isolates (24\%) were traceable back to C. jejuni positive chicken slaughter batches. None of the human isolates were traced back to swimming water, which was rather sporadically sampled. The highly discriminatory wgMLST, together with the patient background information and temporal relationship data with possible sources, offers a new, accurate approach to trace back the origin of domestic campylobacteriosis. Our results suggest that potentially a substantial proportion of campylobacteriosis cases during the seasonal peak most probably are due to other sources than chicken meat consumption. These findings warrant further wgMLST-based studies to reassess the role of other reservoirs in the Campylobacter epidemiology both in Finland and elsewhere.
\end{abstract}

(c) 2016 Elsevier B.V. All rights reserved.

\section{Introduction}

Campylobacteriosis is the most commonly reported cause of bacterial gastroenteritis in the European Union (EU), with 214,779 reported infections in 2013 (EFSA, 2014). In Finland, more than 4251 human Campylobacter infections were registered in 2012 (incidence rate 78.7/ 100000 ) and in 2014 the number of cases increased to 4887 (incidence rate $90.1 / 100000$ ), and approximately $95 \%$ are caused by $C$. jejuni (www.thl.fi). Approximately half of these infections are associated with foreign travel, but in summer, domestically acquired infections increase during the seasonal peak from July to September (Vierikko et al., 2004). Furthermore, most cases are defined as sporadic, which complicates tracing the source of infection (www.thl.fi). Several studies have identified handling of raw or eating improperly cooked poultry meat

\footnotetext{
* Corresponding author at: P.O. Box 66, FI-00014, University of Helsinki, Finland. E-mail address: marja-liisa.hanninen@helsinki.fi (M.-L. Hänninen).
}

as major risk factors for acquiring Campylobacter infection (Levesque et al., 2013; Mughini Gras et al., 2012; Strachan et al., 2013). Other potential sources are the consumption of contaminated beef or unpasteurized milk, drinking water from private wells, and swimming in natural waters, which a previous Finnish case-control study also identified as risk factors (Schönberg-Norio et al., 2004). Furthermore, surface waters have been shown to contain Campylobacter spp. (Hörman et al., 2004) and recreational swimming activities are common in Finland, especially in summer.

C. jejuni is a commensal of the gastrointestinal tract of most warmblooded animals and birds including chicken (http://www.who.int/ mediacentre/factsheets/fs255/en/). Due to EU-legislation, the occurrence of Campylobacter in broiler production has been monitored since 2007 (http://www.efsa.europa.eu/en/topics/topic/campylobacter.htm), revealing a high level of Campylobacter-positive slaughter batches as well as retail meat in several countries. However, the prevalence has remained low in Finland, (EFSA, 2014). Approximately 100 million kg 
of broiler meat is consumed annually in Finland and in 2012, of which the majority originates from domestic production. However, imported chicken meat (90\% from Thailand and Brazil), approximately 1 to 3 million $\mathrm{kg}$, is used in meat processing industry and is therefore not sold as raw meat products at retail (www.mmm.fi). Thus only domestically produced chicken meat contaminated with $C$. jejuni is reasonable to consider as a relevant source to Finnish consumers.

Multilocus sequence typing (MLST) has proved to be an essential tool in studies of the molecular epidemiology and population genetics of C. jejuni (Cody et al., 2013; de Haan et al., 2013; Sheppard et al., 2009). Our previous MLST studies have revealed that the most frequent sequence types (STs) among Finnish C. jejuni isolates, detected in human patients and broiler batches, are ST-45 and ST-230 (ST-45 clonal complex, [CC]), ST-50 (ST-21 CC), ST-267 (ST-283 CC) and ST-677 (ST677 CC) (de Haan et al., 2014; Kovanen et al., 2014; Llarena et al., 2015). Whole-genome sequencing (WGS) and the use of comparative genomics tools such as whole-genome MLST (wgMLST) have become increasingly affordable, providing information from bacterial genomes with a much higher resolution than MLST (Bessell et al., 2012; Cody et al., 2013; Kovanen et al., 2014; Sheppard et al., 2012; Zhang et al., 2015). In combination with other epidemiological data, wgMLST enables the recognition of related isolates, providing more accurate information of the potential sources of infection.

Although several studies have compared $C$. jejuni isolates from the chickens and human patients, the data on temporal relationships between human patient and potential source of infection are rarely included, which would provide valuable knowledge on the epidemiology of the disease. We therefore performed this study (i) to analyze the association of MLST and wgMLST types, using recently developed software for wgMLST (Zhang et al., 2015), of Finnish C. jejuni isolates collected from patients with domestically acquired infections, chicken slaughter batches and swimming water during the seasonal peak in 2012 and (ii) to assess the ability of wgMLST to trace human C. jejuni infections to two different potential sources with simultaneous consideration of their temporal relationships.

\section{Materials and methods}

\subsection{Bacterial isolates}

The human patient $C$. jejuni isolates $(\mathrm{n}=95)$ include all recovered isolates from domestically acquired enteric infections collected during the seasonal peak (June to September) from three hospital districts in Central Finland in 2012 as described in our previous study (Kovanen et al., 2014). The illness was defined as domestic if the patients had not been traveling outside of Finland in two preceding weeks prior to illness. The gender, age and the date of collection of the fecal sample were available to be included in the analysis. C. jejuni was isolated from the fecal samples by direct culture on modified charcoal cefoperazone deoxycholate agar (mCCDA) and confirmed to species level using species specific PCR as described by Kovanen et al. (2014). All clinical and microbiological data was provided by the three clinical laboratories of the districts. The districts center around four cities with populations of 279,000 (district 1), 121,000 (district 2 contains two cities), and 265,000 (district 3) inhabitants (http://www.stat.fi/tup/tilastotietokannat/ index_en.html).

The included chicken-derived C. jejuni isolates $(n=83)$ were collected during 2012 in the Finnish Campylobacter monitoring program for poultry (Maa- ja Metsätalousministeriö, 2007) and are described in more detail in our previous study (Llarena et al., 2015). In brief, the surveillance program sampled all chicken batches slaughtered in Finland between June and October, and the detection of $C$. jejuni was done by direct plating of a pooled cecal sample ( 10 cecas/batch) on mCCDA. A single typical colony was selected for further analysis according to the method of The Food Safety Authority (Evira) 3512/5 (The Finnish Food Safety Authority Evira, 2012). Of the total 83 isolates included in the study (83 positive slaughter batches), 75 were isolated during the same period as the human patient isolates (June to September) and originated from a total of 37 farms indicating that a positive farm could have several positive batches (from one to four). According to the National Report to the European Food Safety Authority (EFSA), the total number of studied slaughter batches during the national reporting period from June to October was 1534 , revealing that only $5.3 \%$ of all slaughter batches were positive for $C$. jejuni 2012 (EFSA, 2014; Llarena et al., 2015). Thus, the isolates represented $96.3 \%$ of the total C. jejuni population detected during the study period. Eight of the total 83 C. jejuni slaughter batch isolates were outside of the seasonal peak and were collected either in March, May or October. All 83 isolates were included in the wgMLST analysis. Fifty one of the total of 83 isolates represented single STs from the farms and the remaining 32 isolates were from the successive slaughter batches reared simultaneously on the farms. They had similar STs and PFGE types within successive batches. These 32 isolates were therefore excluded in the descriptive statistics analysis to avoid overestimating ST frequencies, as described by Llarena et al. (2015).

In addition to these, $20 \mathrm{C}$. jejuni isolates (from a total of 50 samples) from recreational swimming beaches (12 on lakes and one on a river) were collected from June to August 2012. Seven of the isolates were collected from $11 \mathrm{EU}$ beaches, each sampled once a month (EU directive: 2006/7/EY, Ministry of Social affairs and Health: 711/2014) and located in the same three districts where the human isolates were collected. In addition, 13 isolates originated from samples collected approximately twice a month from a lake and a river located in the Helsinki metropolitan area (used as controls). Samples (100 $\mathrm{ml}$ or subsequent volumes of $100 \mathrm{ml}$ and $1.5 \mathrm{l}$, stored at $+4{ }^{\circ} \mathrm{C}$ prior to analysis) were concentrated using $0.45 \mu \mathrm{m}$ pore size membrane filters (Millipore, Billerica, MA, USA), enriched in $100 \mathrm{ml}$ of Bolton enrichment broth (Oxoid Ltd., Basingstoke, Hampshire, UK) including $5 \%$ of defibrinated horse blood and incubated microaerobically at $+37^{\circ} \mathrm{C}$ for $48 \mathrm{~h}$. Ten $\mu \mathrm{l}$ was streaked onto mCCDA plates (Oxoid Ltd.) and incubated microaerobically at $41.5 \pm 0.5^{\circ} \mathrm{C}$ for 24-48 h. Typical colonies were stored in nutrient broth (85\% Nutrient broth, $15 \%$ glyserol) at $-70{ }^{\circ} \mathrm{C}$. DNA was extracted using the Wizard genomic DNA purification kit (Promega, Mannheim, Germany) and species (C. jejuni) was confirmed using multiplex PCR (Kovanen et al., 2014).

\subsection{Whole-genome sequencing}

Draft genome sequences were determined using Illumina HiSeq sequencing technology (Nextera library, 100 cycles, paired-end library, $>40 \times$ coverage). NGS library preparation, enrichment, sequencing and sequence analysis were performed by the Institute for Molecular Medicine Finland (FIMM Technology Centre, University of Helsinki, Finland). Reads were assembled using SPAdes 3.2.1 (Bankevich et al., 2012) using default settings.

\subsection{MLST and wgMLST}

MLST types were assigned using the Campylobacter MLST database (http://pubmlst.org/campylobacter/). Assembled contigs of the wholegenome sequences were further analysed applying a gene-by-gene approach using Genome profiler (GeP) (Zhang et al., 2015). The draft genomes of four isolates (three human and one water isolate) were too fragmented (> 100 contigs) and were excluded from further wgMLST analysis. The Split Decomposition networks, representing allelic distance matrix of the shared loci of the isolates, were constructed for each ST using SplitsTree 4 (Huson and Bryant, 2006) and edited using CorelDRAW X6 (Corel Corporation, Ottawa, Ontario, Canada). To increase resolution, isolates forming clusters within the same ST were re-analysed using GeP. To identify single nucleotide polymorphisms (SNPs), alignments of loci having multiple allele types were manually inspected. Variations in homopolymeric tract lengths were not considered as SNPs (Bayliss et al., 2012), even though they were registered 
in the total number of allele differences observed between the isolates in the $\mathrm{GeP}$ analysis. Since previous studies have shown that a few single nucleotide polymorphisms (SNPs) occur during passage through the host or within an outbreak (Revez et al., 2013, 2014), herein $\leq 5$ SNPs between the genomes were used as a cut-off value to indicate genetically highly related isolates that were considered representative of the same clone.

\subsection{Data deposition}

The draft genomes of all isolates used in wgMLST analysis were deposited to the PubMLST database (http://pubmlst.org/campylobacter/).

\subsection{Temporal relationship of chicken and human isolates}

In tracing the patient $C$. jejuni isolates to chicken slaughter batches we used the following temporary relationship estimations: the chicken isolates originating from the positive slaughter batches were considered a potential source of human infection if the slaughter preceded the illness by two to 23 days (the time between the date of slaughter and of the patient sampling), a period that takes into consideration the time the chicken products go on sale and the incubation time of the illness as described previously (Kärenlampi et al., 2003).

\section{Results}

3.1. MLST and the temporal relationship between Finnish human, chicken and swimming water $C$. jejuni isolates

The distribution of STs among human, chicken and water $C$. jejuni isolates is shown in Fig. 1. The most frequent STs were ST-45 and ST230 (ST-45 CC), ST-267 (ST-283 CC) and ST-677 (ST-677 CC) covering a total of $75 \%$ of the human and $64 \%$ of the chicken isolates (Fig. 1). In addition, ST-11 (ST-45 CC) was detected in 14\% of the chicken isolates but in only one patient; and two ST-794 (ST-677 CC) isolates were detected in both chicken and human isolates. In total, $79 \%$ of the STs from human isolates overlapped with the chicken STs (Fig. 1). Furthermore, $50 \%$ of the swimming water isolates $(n=20)$ were either ST-45 ( $30 \%$ of the isolates), ST-230 (15\% of the isolates) or ST-677 (5\% of the isolates) (Fig. 1). Four out of seven swimming water isolates (57\%), collected from the same three districts as the human isolates had overlapping STs. Other, unique STs that did not overlap between human, chicken and water isolates accounted for $20 \%, 17 \%$ and $45 \%$ of the human, chicken and water isolates, respectively (Fig. 1).
After taking into account the time between the isolation of $C$. jejuni from the chicken slaughter batches and the patient sampling, the overlap between human and chicken STs decreased from $79 \%$ to $48 \%$. Weekly isolation data for the five most common STs (ST-45, ST-230, ST-267, ST-677 and ST-11) from different sources are shown in Fig. S1 (Supplementary data, Fig. S1). Among ST-45, ST-230 and ST-267, most chicken (82.5\%) and human (92.4\%) isolates were detected from the middle of July to the middle of August. However, most of the swimming water isolates of ST-45, ST-230 and ST-677 (71.4\%) were detected prior to the positive chicken slaughter batches and human infections from June to the middle of July. All human ST-677 isolates were detected several weeks earlier or during the same week as the ST-677 positive chicken slaughter batches (Fig. S1). In week 32 (Fig. S1), however, two human isolates were detected three days after the slaughter of the positive chicken batch revealing the only potential temporal overlap between the chicken and human ST-677 isolates.

\subsection{WgMLST analysis and temporal association between human and chick- en isolates}

With the increased resolution achieved using wgMLST, genetically related $C$. jejuni isolates among human patients and chicken batches were found. The Split Decomposition networks of the five most common STs are shown in Fig. 2 (ST-45 and ST-230) and Fig. 3 (ST-267, ST-11 and ST-677). According to our wgMLST analysis, 22 of the total of 92 human C. jejuni isolates (24\%) could be both temporally (223 days after slaughter of $C$. jejuni positive chicken batch) and genetically ( $\leq 5$ SNPs) traced to a chicken isolate. The sampling dates and allelic differences of the genetically related isolates are described in detail in Table 1. Most of the allelic variation was identified as changes in homopolymeric track lengths (Table 1 ).

ST-45 isolates formed three distinct clusters that differed from each other by $400-550$ alleles (Fig. 2A). With our criteria, three human isolates in Cluster 1 (J5, J12 and J13) and one in Cluster 2 (J11) could be temporally and genetically traced back to a chicken isolate (Cluster 1: chicken isolate 5913 and Cluster 2: chicken isolate 6179; Fig. 2A). Details of the dates of isolation and genomic differences (i.e., SNPs) between these chicken and human isolates are represented in Table 1. Temporally and genetically linked human and chicken isolates showed 1-3 SNPs in Cluster 1 and 1 SNP in Cluster 2 (Fig. 2A, Table 1) based on GeP analysis. Even though Cluster 3 contained 13 chicken batch isolates and four patient isolates they differed by approximately 50 to 70 alleles from each other (Fig. 2A). Further, Cluster 3 contained chicken isolates 1994 and 1995 (two batches from the same farm); 6497 and

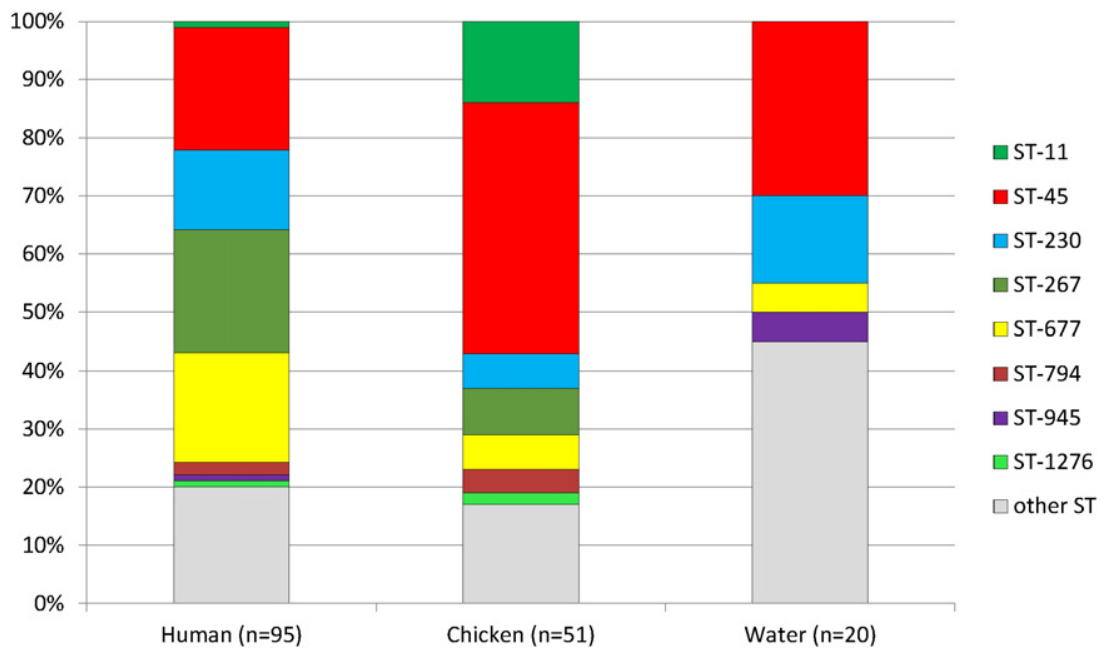

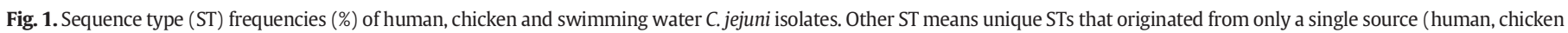
or swimming water) and thus did not overlap between sources. 


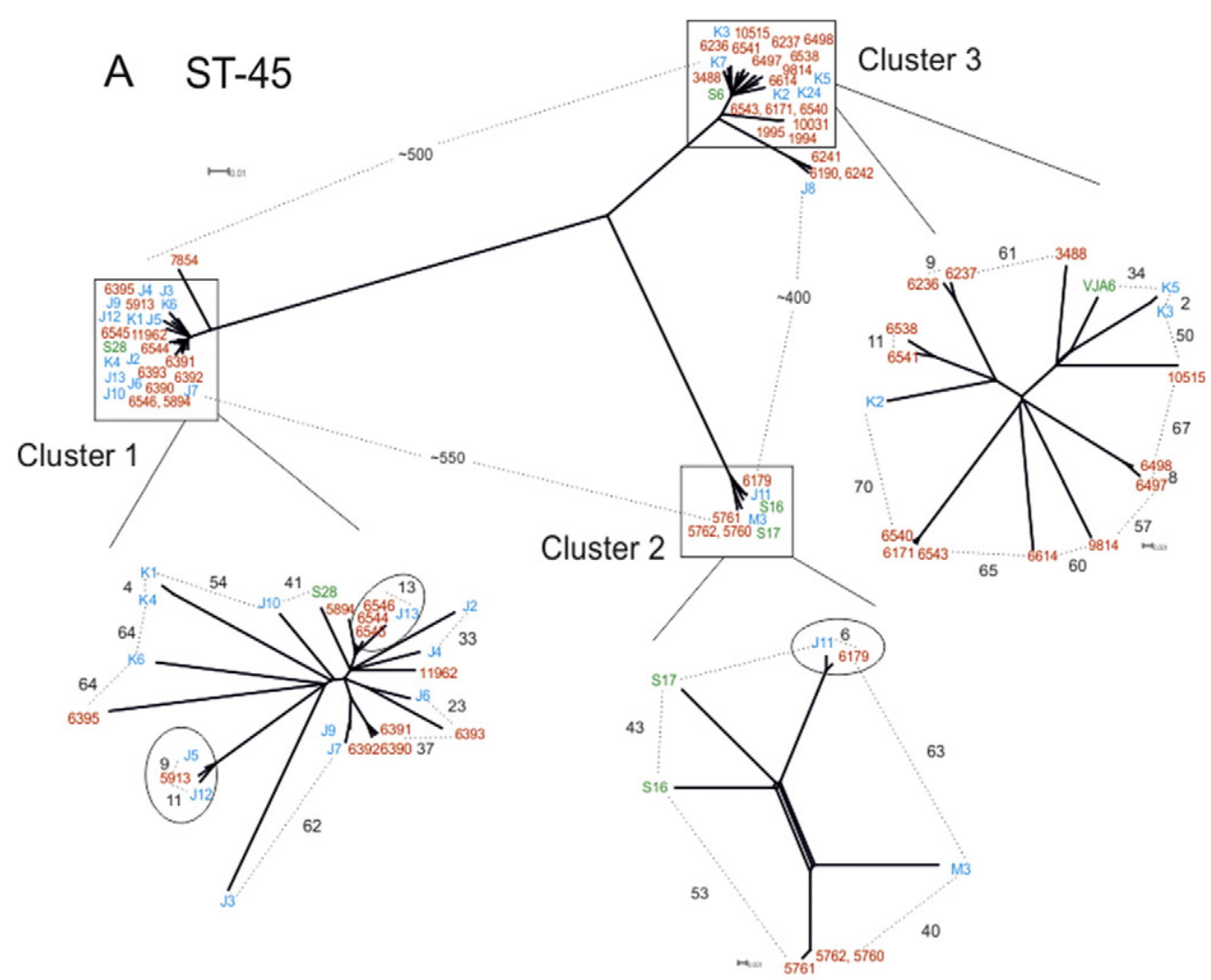

B ST-230

Cluster 4

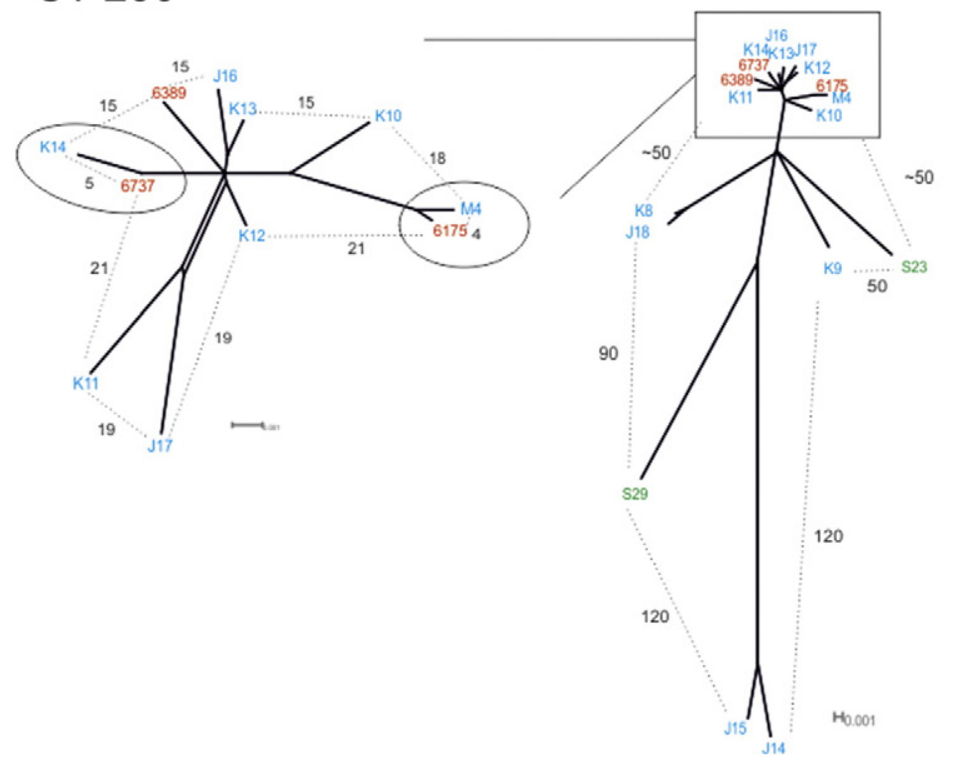

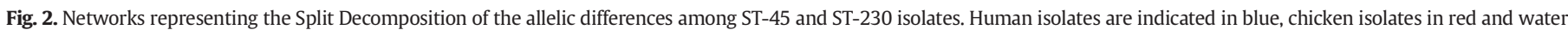

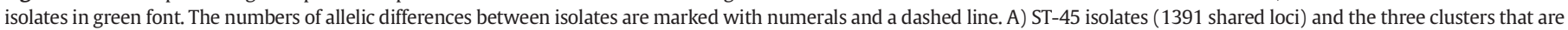

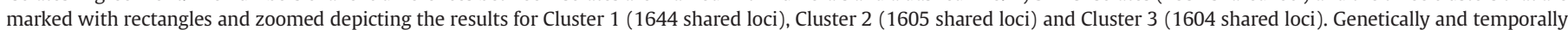

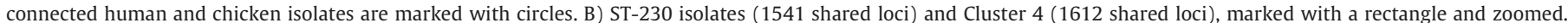
Genetically and temporally connected human and chicken isolates are marked with circles.

6498 (two batches from the same farm); and 6236 and 6237 (two batches from the same farm), differing from each other by only 0-5 SNPs. However, four chicken isolates (6538, 6541, 6540 and 6543; Fig. 2A), also located in Cluster 3 and originating from different batches of the same farm, were of two clones that differed by $~ 70$ alleles.

Two human ST-230 isolates in Cluster 4 were genetically (wgMLST) and temporally traced to chicken isolates, containing two (M4 and
6175, Fig. 2B) and one (K14 and 6737, Fig. 2B) SNPs (Table 1). Among the ST-267 isolates in Cluster 5, only 0-4 SNPs separated a human and chicken isolates. Considering the temporal relationship between the isolates, the 15 out of 18 human ST-267 isolates could be linked to chickens (Cluster 5 in Fig. 3, Table 1).

One ST-11 human isolate (J1) was temporally and genetically linked with a chicken isolate (Cluster 6, Fig. 3, Table 1). None of the human ST- 


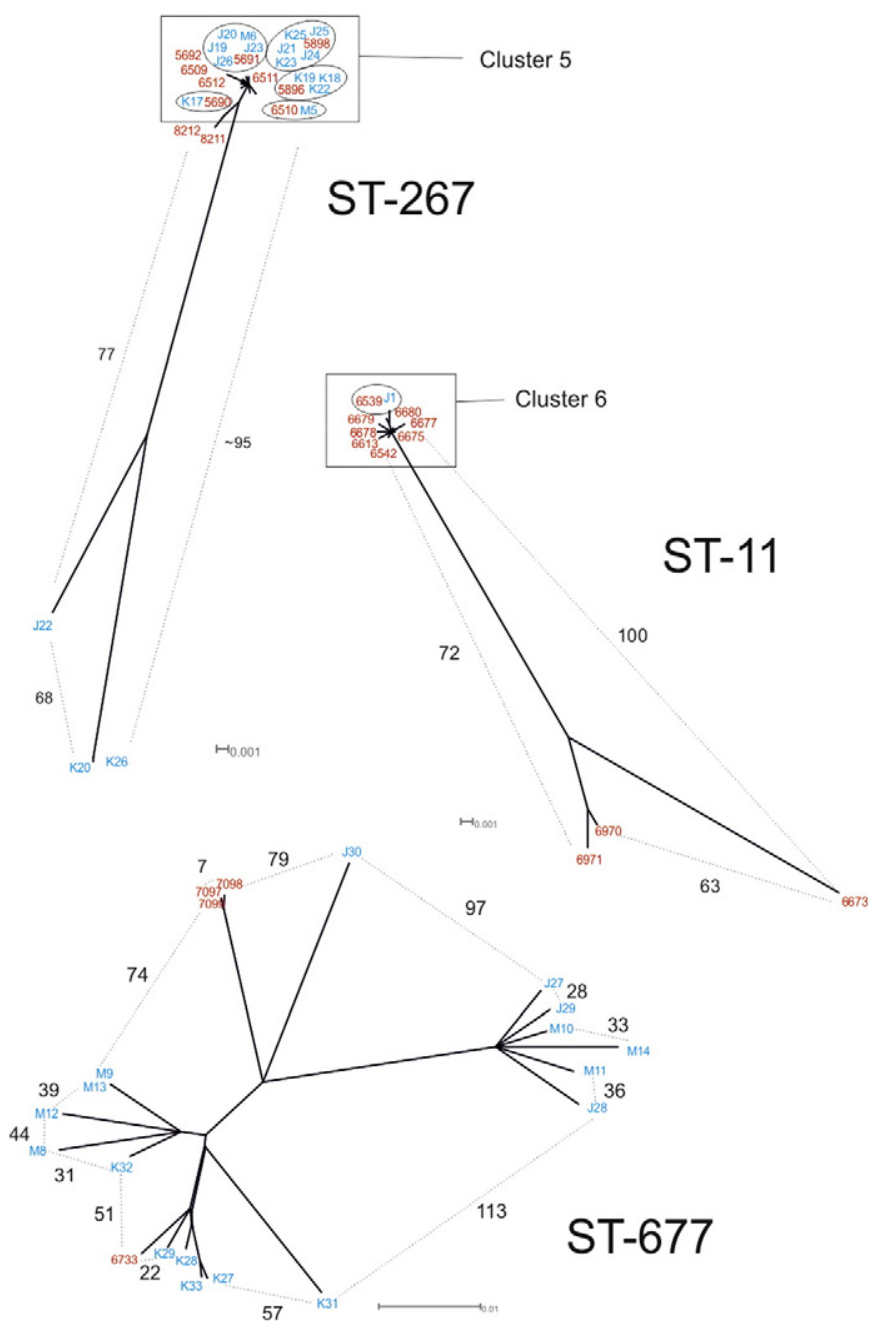

Fig. 3. Networks representing the Split Decomposition of the allele differences among ST267 (1560 shared loci), ST-11 (1605 shared loci) and ST-677 (1618 shared loci) isolates. Human isolates are indicated in blue and chicken isolates in red font. Cluster 5 (1566 shared loci) and Cluster 6 (1615 shared loci) isolates are marked with rectangles and temporally and genetically associated human and chicken isolates are marked with circles. Allelic differences between isolates are marked with numerals and a dashed line.

677 isolates could be traced to a chicken isolates (Fig. 3), either genetically (allelic differences ranging from 51 to 79 ) or temporally, since most of the human ST-677 isolates were detected before the positive chicken slaughter batches. Three of ST-677 chicken isolates were highly similar and two of them (7098 and 7099) originated from two slaughter batches from the same farm (Table S1). Similarly, other minor STs that overlapped between human and chicken isolates (ST-794 and ST1276) or human and water isolates (ST-945) revealed no temporal or close genetic relationship (data not shown). Further, eight $C$. jejuni chicken isolates outside the major study season were used as controls for discriminatory power of wgMLST. None of them were genetically highly related to any of the human isolates.

\section{3. wgMLST similarity of the isolates between slaughter batches from the same farms}

We had a total of 11 farms which produced several C. jejuni positive slaughter batches (two to four batches) during a rearing cycle. A list of the isolates from successive slaughter batches is presented in Table S1.Within a farm, all isolates from different slaughter batches were of a single MLST type and their wgMLST types were located in same clusters in the Split Decomposition analysis, differing only by a few SNPs (0-5 SNPs) (Fig. 2A, Table S1). Detailed wgMLST results are presented in 3.2.

\section{Discussion}

Although Campylobacter jejuni has been a well-identified cause of bacterial gastroenteritis for over three decades (Skirrow, 1977), the epidemiology of the disease, including the relative significance of different sources and transmission routes of the bacteria, remains unclear (Colles and Maiden, 2012) and might have regional variations. EFSA Panel of Biological Hazards (BIOHAZ) has estimated that $50-80 \%$ of human Campylobacter infections as a whole (including both reservoir and source) are of chicken origin (EFSA, 2011) and the present study also revealed a major $79 \%$ overlap among the MLST types of human and chicken isolates. The high genotype overlap between human patient and chicken strains suggests that chicken meat production as one of the major reservoirs for $C$. jejuni, facilitating amplification of different $C$. jejuni genotypes. Similar results have been obtained in previous studies, based merely on the MLST similarity between the chicken and human isolates (de Haan et al., 2014; Sheppard et al., 2009) or in source attribution modeling studies, which have attributed from $45.4 \%$ (de Haan et al., 2013), via $64.5 \%$ (Levesque et al., 2013) to 97\% (Wilson et al., 2008) of the human isolates to a chicken origin. Since chicken production systems and human meat consumption habits vary between countries, the relative proportion of campylobacteriosis cases due to chicken meat consumption will also differ between regions (EFSA, 2011). In the present study, taking into account information about the temporal relationship when tracing isolates from human domestic infections from three districts to chicken slaughter batches, we found a MLST overlap of $48 \%$. This result further confirms the results from two independent, previous Finnish studies where the overlap between chicken and human $C$. jejuni isolates decreased from $69.8 \%$ similarity of PFGE types to $31.4 \%$ (Hakkinen et al., 2009), and from $46 \%$ similarity of combined Penner-serotypes and PFGE types to 31\% (Kärenlampi et al., 2003), when accounting for the isolation time of the patient fecal sample and slaughter day of the chicken batch. Further comparison of the isolates at the wgMLST level and their temporal relationship, as done in this study, revealed that $24 \%$ of the human infections could be traced back to chicken slaughter batches. This result diverges remarkably from the overall MLST overlap mentioned above, but is, however, concordant with the BIOHAZ panel's estimation that the direct exposure from handling and consumption of broiler meat may account for 20-30\% of human infections (EFSA, 2011).

The rather low overlap between chicken and human isolates is supported by the structure of the Finnish chicken meat production and management systems that only infrequently produce Campylobacterpositive chicken flocks. EU member states annually monitor and report Campylobacter findings on the chicken production chain to EFSA. In 2012, the prevalence of Campylobacter positive slaughter batches were, for example, $47 \%$ in Austria and $62 \%$ in Spain, but in Finland, only $5.3 \%$ of all produced batches were tested positive during the summer peak season, and 1.6\% during the winter (EFSA, 2014), and the annual prevalence has remained constantly low (Llarena et al., 2015). The low prevalence may be associated with the climate, adequate biosecurity and close collaborations between farmers and slaughterhouses (Llarena et al., 2015). In addition, as a previous European-wide study containing data from several countries showed, Finnish broiler carcasses carry low counts of Campylobacter $(97.8 \%<10 \mathrm{CFU} / \mathrm{g}$ ) (EFSA, 2010). Furthermore, most chicken meat sold at retail are packed in modified atmosphere and the products are often marinated and therefore require no further handling before cooking at home, decreasing the risk of cross-contamination (Gonzalez and Hänninen, 2011). All these facts support a low exposure probability from chicken meat in Finland. In addition, our findings are in line with the estimated low risk per serving of Finnish broiler meat for contracting campylobacteriosis as reported by EFSA BIOHAZ Panel (2011). 
Table 1

Genetic variation of temporally and genetically associated chicken and human isolates.

\begin{tabular}{|c|c|c|c|c|c|c|}
\hline \multicolumn{7}{|l|}{ ST-45 } \\
\hline Cluster 1 (1644 loci) & Source & Sampling date & Time difference & Genomic differences & SNPS & Amino acid change \\
\hline 5913 & Chicken & 16/Jul/2012 & & & & \\
\hline \multirow[t]{3}{*}{$\mathrm{J} 12$} & Human & 18/Jul/2012 & 2 days & Hypothetical protein & $\mathrm{T} \rightarrow \mathrm{A}$ & stop -> K \\
\hline & & & & ABC-type tungstate transport system, permease protein & $\mathrm{C} \rightarrow \mathrm{T}$ & no change \\
\hline & & & & oligopeptide transporter ${ }^{\mathrm{a}}$ & $\mathrm{T}->-$ & $\mathrm{N}->$ stop \\
\hline J5 & Human & 26/Jul/2012 & 10 days & Hypothetical protein ${ }^{\mathrm{a}}$ & $\mathrm{T} \rightarrow \mathrm{A}$ & stop $->\mathrm{K}$ \\
\hline 6544 & Chicken & 31/Jul/2012 & & & & \\
\hline \multirow[t]{5}{*}{$\mathrm{J} 13$} & Human & 14/Aug/2012 & 14 days & Transcriptional repressor of $\mathrm{CmeABC}$ operon & $\mathrm{T} \rightarrow>-->\mathrm{A}$ & Y -> stop stop -> E \\
\hline & & & & Recombination protein RecR & $\mathrm{G}->\mathrm{A}$ & $\mathrm{G}->\mathrm{E}$ \\
\hline & & & & Sulfate adenylyltransferase subunit 1 & $\mathrm{C}->\mathrm{T}$ & no change \\
\hline & & & & Methionine aminopeptidase & $C \rightarrow A$ & $\mathrm{~L}->\mathrm{I}$ \\
\hline & & & & $\mathrm{Fe} 2+\mathrm{ABC}$ transporter, substrate binding protein ${ }^{\mathrm{a}}$ & $\mathrm{T}->\mathrm{G}$ & $\mathrm{I}->\mathrm{M}$ \\
\hline
\end{tabular}

\begin{tabular}{|c|c|c|c|c|c|c|}
\hline Cluster 2 (1605 loci) & Source & Sampling date & Time difference & Genomic differences & SNPs & Amino acid change \\
\hline 6179 & Chicken & 19/Jul/2012 & & & & \\
\hline J11 & Human & 2/Aug/2012 & 14 days & Magnesium and cobalt transport protein $\operatorname{Cor}^{\mathrm{a}}$ & $C->A$ & $\mathrm{~T} \rightarrow \mathrm{K}$ \\
\hline \multicolumn{7}{|l|}{ ST-230 } \\
\hline Cluster 4 (1612 loci) & Source & Sampling date & Time difference & Genomic differences & SNPs & Amino acid change \\
\hline 6175 & Chicken & 20/Jul/2012 & & & & \\
\hline \multirow{2}{*}{ M4 } & Human & 10/Aug/2012 & 21 days & Cytolethal distending toxin subunit C & $G \rightarrow A$ & $A->T$ \\
\hline & & & & Type II secretion system protein ${ }^{a}$ & $\mathrm{C}->\mathrm{T}$ & $\mathrm{F}->\mathrm{S}$ \\
\hline 6737 & Chicken & 9/Aug/2012 & & & & \\
\hline K14 & Human & 20/Aug/2012 & 11 days & Hypothetical protein $^{\mathrm{a}}$ & $\mathrm{T}->\mathrm{G}$ & $V->G$ \\
\hline
\end{tabular}

ST-267

\begin{tabular}{|c|c|c|c|c|c|c|}
\hline Cluster 5 (1566 loci) & Source & Sampling date & Time difference & Genomic differences & SNPS & Amino acid change \\
\hline 5690 & Chicken & 6/Jul/2012 & & & & \\
\hline K17 & Human & 20/Jul/2012 & 14 days & $\begin{array}{l}\text { Membrane protein related to metalloendopeptidases } \\
\text { Hypothetical protein }^{\mathrm{a}}\end{array}$ & $\begin{array}{l}A->T \\
T->\text { G poly } G(-/ G)\end{array}$ & \multirow[t]{2}{*}{$\begin{array}{l}\mathrm{K}->\mathrm{I} \\
\text { stop }->\mathrm{R} \text { V }->\mathrm{G}\end{array}$} \\
\hline 5691 & Chicken & 5/Jul/2012 & & & & \\
\hline $\mathrm{J} 26$ & Human & $22 / \mathrm{Jul} / 2012$ & 17 days & a & & \\
\hline M6 & Human & 13/Jul/2012 & 8 days & \multirow{2}{*}{$\begin{array}{l}\text { Capsular polysaccharide } \mathrm{ABC} \text { transporter }{ }^{\mathrm{a}} \\
\mathrm{a}\end{array}$} & $G->A$ & \multirow[t]{2}{*}{$E->K$} \\
\hline J19 & Human & 19/Jul/2012 & 14 days & & & \\
\hline $\mathrm{J} 20$ & Human & 26/Jul/2012 & 21 days & \multirow{2}{*}{$\begin{array}{l}\text { Capsular polysaccharide } \mathrm{ABC} \text { transporter }^{\mathrm{a}} \\
\text { a }\end{array}$} & \multirow[t]{2}{*}{$G \rightarrow A$} & \multirow[t]{3}{*}{$\mathrm{E}->\mathrm{K}$} \\
\hline $\mathrm{J} 23$ & Human & 25/Jul/2012 & 20 days & & & \\
\hline 5896 & Chicken & 11/Jul/2012 & & & & \\
\hline \multirow[t]{2}{*}{ K18 } & \multirow[t]{2}{*}{ Human } & \multirow[t]{2}{*}{ 25/Jul/2012 } & \multirow[t]{2}{*}{14 days } & $\begin{array}{l}\text { hypothetical protein } \\
\text { Dihydroneopterin aldolase }\end{array}$ & $\mathrm{T} \rightarrow \mathrm{G}$ & \multirow{2}{*}{$\begin{array}{l}V->G \\
E->G\end{array}$} \\
\hline & & & & Dihydroneopterin aldolase $^{a}$ & $A \rightarrow G$ & \\
\hline \multirow[t]{2}{*}{ K19 } & \multirow[t]{2}{*}{ Human } & \multirow[t]{2}{*}{ 17/Jul/2012 } & \multirow[t]{2}{*}{6 days } & \multirow{2}{*}{$\begin{array}{l}\text { Dihydroneopterin aldolase } \\
\text { Rod shape-determining protein } \mathrm{MreB}^{\mathrm{a}}\end{array}$} & $A \rightarrow G$ & \multirow{2}{*}{$\begin{array}{l}\mathrm{E} \rightarrow \mathrm{G} \\
\mathrm{T} \rightarrow \mathrm{I}\end{array}$} \\
\hline & & & & & $\mathrm{C} \rightarrow \mathrm{T}$ & \\
\hline \multirow[t]{2}{*}{ K22 } & \multirow[t]{2}{*}{ Human } & 19/Jul/2012 & 8 days & Alcohol dehydrogenase & TAA $->$ TCT & stop $->$ S \\
\hline & & & & Dihydroneopterin aldolase & $A->G$ & $E->G$ \\
\hline 5898 & Chicken & 11/Jul/2012 & & & & \\
\hline $\mathrm{J} 21$ & Human & 25/Jul/2012 & 14 days & Ribose-phosphate pyrophosphokinase ${ }^{a}$ & $\mathrm{~T} \rightarrow \mathrm{C}$ & no change \\
\hline $\mathrm{J} 24$ & Human & 2/Aug/2012 & 22 days & a & & \\
\hline $\mathrm{J} 25$ & Human & 30/Jul/2012 & 19 days & Ribose-phosphate pyrophosphokinase & $\mathrm{T} \rightarrow \mathrm{C}$ & no change \\
\hline & & & & Putative oxidoreductase ferredoxin-type protein ${ }^{a}$ & $A \rightarrow G$ & $\mathrm{~T}->\mathrm{A}$ \\
\hline K23 & Human & 19/Jul/2012 & 8 days & Ribose-phosphate pyrophosphokinase $\mathrm{a}^{\mathrm{a}}$ & $\mathrm{T} \rightarrow \mathrm{C}$ & no change \\
\hline K25 & Human & 23/Jul/2012 & 12 days & Ribose-phosphate pyrophosphokinase ${ }^{a}$ & $\mathrm{~T} \rightarrow \mathrm{C}$ & no change \\
\hline 6510 & Chicken & 28/Jul/2012 & & & & \\
\hline M5 & Human & 11/Aug/2012 & 14 days & a & & \\
\hline ST-11 & & & & & & \\
\hline Cluster 6 (1615 loci) & Source & Sampling date & Time differ & Genomic differences & SNPs & Amino acid change \\
\hline 6539 & Chicken & 31/Jul/2012 & & & & \\
\hline $\mathrm{J} 1$ & Human & 13/Aug/2012 & 13 days & Imidazole glycerol phosphate synthase cyclase & ubunit $^{\mathrm{a}}$ & $\mathrm{S}->\mathrm{L}$ \\
\hline
\end{tabular}

${ }^{a}$ Changes in homopolymeric tracts (poly G or poly C).

For the first time the use of whole genome sequencing was applied to trace human campylobacteriosis back to chicken batches. This was possible due to the highly sensitive nature of wgMLST, and the use of a newly developed genomic analysis tool GeP (Zhang et al., 2015), which allowed sequence comparisons of 1391 to 1644 shared loci of the studied isolates within a ST, compared to sequences of seven loci used in traditional MLST. Broader use of wgMLST combined with epidemiological data, for example, site and time of infection (domestic/foreign travel-associated) and site-relatable data from potential source(s), will provide more precise estimates of the role of chicken meat or other sources in different geographical areas and will substantially improve our understanding of the epidemiology of human $C$. jejuni infections.

Our wgMLST results from successive slaughter batches reared simultaneously at farms indicated that chickens were contaminated with a single wgMLST genotype only (except a case when the isolates of ST45 had 70 allelic differences). However, it is possible that some other 
minor STs may have been present in the batches, but were not detected due to the sampling procedure, since only one colony was taken for further analysis, thereby resulting in an underestimation of the overlap between human and chicken isolates. However, we consider this possibility as minor due to several reasons. First, the robustness of the sampling procedure ensued the inclusion of $>95 \%$ of the chicken isolates derived from the monitoring program in 2012. This isolate collection is representative of the $C$. jejuni population circulating among Finnish chicken flocks during the seasonal peak of 2012 and depicts well the campylobacteriosis risk posed to the Finnish consumers through chicken meat consumption. Further, unpublished Finnish studies have shown that once Campylobacter colonization is detected in a chicken flock, the majority of the birds are colonized by a single PFGE type (Hakkinen and Kaukonen, 2009, presented at the 15th International Workshop on Campylobacter, Helicobacter and Related Organisms, Niigata, Japan, $2-9$, September). In addition, according to the findings of Llarena et al. (2015), the $C$. jejuni isolates collected from different flocks raised simultaneously were of the same PFGE type on the majority of occasions (76.3\%) during a study period of five years. Taken together, the inclusion of one isolate from each $C$. jejuni-positive chicken batch was therefore considered as adequate for estimating the genotype in a chicken flock.

We have previously used the $\leq 5$ SNPs cut-off value to associate outbreak-associated $C$. jejuni isolates and separating them from control isolates (Revez et al., 2014). In the present study, we found this cut-off value suitable to identify clustering $C$. jejuni isolates from successive slaughter batches and define genetically closely related chicken and human isolates. The selected cut-off value was also supported by the findings that no human and chicken isolates differed by 6-9 SNPs, which would make the chosen cut-off value less suitable. However, in two cases, 10 SNP difference was found between a human and chicken ST-230 isolates, which also had a temporal connection. Ten to fourteen SNPs differences were also detected in five other cases, but these isolates were not temporally associated, therefore excluding them from the scheme. In other comparisons, the SNP differences were higher (>15 SNPs). In addition to SNPs, we commonly found variations in the lengths of homopolymeric tracts (poly $\mathrm{G}$ or poly $\mathrm{C}$ tracts) in phasevariable genes (Bayliss et al., 2012), which is in line with previous reports (Revez et al., 2014).

ST-677 was detected in both human and chicken isolates, but the peak of ST-677 in human infections occurred in July. A similar July-peak of ST677 in human infections was also seen in Finland in a previous study (de Haan et al., 2014), whereas $80 \%$ of the chicken ST-677 isolates collected in five years were detected in August or September, suggesting persistence of seasonality among ST-677 (Llarena et al., 2015). In the present study, almost all chicken ST-677 isolates were detected several weeks after isolation from the human patients, suggesting that ST-677, a common ST among Finnish clinical isolates, transmits to humans from elsewhere, probably environmental sources. A common source for humans and chickens is supported by the fact that the chicken flocks usually become contaminated during the first weeks of the rearing period (Hermans et al., 2012). However, the chicken and human ST-677 isolates diverged at the wgMLST level suggesting that the reservoir(s) infecting chicken flocks and human patients could also be different.

Interestingly, most of the human ST-267 isolates (15 out of 18) were genetically highly related to ST-267 chicken isolates with only 0-4 SNP differences among the 1560 shared loci analyzed in wgMLST. Also the nine chicken isolates were similar to each other, even though they originated from three different farms suggesting that certain closely related genotypes were widely spread that time. In addition to the close genetic relationships, the slaughter dates of the chicken batches preceded the human infections, thereby indicating a temporal relationship. These results suggest that these isolates formed a highly clonal sub-lineage inside ST-267, which seemed to efficiently infect patients. In our previous studies this ST has also been detected both from chickens and human patients (de Haan et al., 2014). Further genomic level and epidemiological studies are warranted to better understand the ecology of this ST.
Three common STs detected in human patients (ST-45, ST-230 and ST-677) were also found in swimming water isolates, suggesting the wide presence of these STs also in environment. However, because none of the C. jejuni isolates from our swimming water samples were genetically closely related with any of the human isolates, we were unable to confirm the results of a previous case-control study that identified swimming in natural waters as a significant risk factor for Campylobacter infection during the seasonal peak in July or August (Schönberg-Norio et al., 2004). One reason for our result is the complexity of environmental sampling. Our sampling was performed by the municipal public health authority and consisted of monthly sampling according to EU legislation (EU directive: 2006/7/EY, Ministry of Social affairs and Health: 711/2014). To increase the possibility of detecting isolates, which could be associated with human infections, the sampling should be much more intensive, continuous and cover more sampling sites to properly examine the role of swimming water in human campylobacteriosis.

\section{Conclusions}

Our studies confirmed the results of several previous studies revealing a high overlap of MLST types between chicken and human isolates and indicated that chicken meat production is a major reservoir of C. jejuni. In addition, using highly discriminatory wgMLST combined with temporal relationship between patient illness and chicken slaughter we were able to trace $24 \%$ of the human C. jejuni isolates back to chicken slaughter batches in July-August 2012. The results showed the power of the whole genome analysis in reconstructing the chicken slaughter batch-associated transmission route of the infection for human domestically acquired campylobacteriosis. Further, our study pointed out that for more successful public health intervention strategies there is a need to identify other potential transmission routes involved in domestically acquired campylobacteriosis, especially in a country with very low prevalence of positive chicken flocks. Furthermore, the results showed that the high discriminatory power of wgMLST supports its use as an accurate method for comparison of temporarily associated isolates. The method was able to identify epidemiologically related, successive $C$. jejuni isolates at farms and distinguish them from nonrelated isolates. However, further studies on the genomic stability and interpretation of genetic changes between the isolates are needed.

Supplementary data to this article can be found online at http://dx. doi.org/10.1016/j.ijfoodmicro.2016.03.009.

\section{Acknowledgements}

We thank the infectious disease specialists of the hospital districts, the municipal health authorities that supplied the water samples and EVIRA for their collaboration. This study was funded by the Academy of Finland with grants on behalf of CoE-MiFoSa (no. 11411405) and Ministry of Agriculture and Forestry (MMM/776/312/2012, EMIDA ERA-NET CamChain). This publication made use of the pubMLST.org/ campylobacter website (Jolley and Maiden, 2010).

\section{References}

Bankevich, A., Nurk, S., Antipov, D., Gurevich, A.A., Dvorkin, M., Kulikov, A.S., Lesin, V.M., Nikolenko, S.I., Pham, S., Prjibelski, A.D., Pyshkin, A.V., Sirotkin, A.V., Vyahhi, N., Tesler, G., Alekseyev, M.A., Pevzner, P.A., 2012. SPAdes: a new genome assembly algorithm and its applications to single-cell sequencing. J. Comput. Biol. 19, 455-477.

Bayliss, C.D., Bidmos, F.A., Anjum, A., Manchev, V.T., Richards, R.L., Grossier, J.P., Wooldridge, K.G., Ketley, J.M., Barrow, P.A., Jones, M.A., Tretyakov, M.V., 2012. Phase variable genes of Campylobacter jejuni exhibit high mutation rates and specific mutational patterns but mutability is not the major determinant of population structure during host colonization. Nucleic Acids Res, 40, 5876-5889.

Bessell, P.R., Rotariu, O., Innocent, G.T., Smith-Palmer, A., Strachan, N.J.C., Forbes, K.J., Cowden, J.M., Reid, S.W.J., Matthews, L., 2012. Using sequence data to identify alternative routes and risk of infection: a case-study of Campylobacter in Scotland. BMC Infect. Dis. 12 (80). http://dx.doi.org/10.1186/1471-2334-12-80 (12 2012). 
Cody, A.J., McCarthy, N.D., Van Rensburg, M.J., Isinkaye, T., Bentley, S.D., Parkhill, J., Dingle, K.E., Bowler, I.C.J.W., Jolley, K.A., Maiden, M.C.J., 2013. Real-time genomic epidemiological evaluation of human Campylobacter isolates by use of whole-genome multilocus sequence typing. J. Clin. Microbiol. 51, 2526-2534.

Colles, F.M., Maiden, M.C., 2012. Campylobacter sequence typing databases: applications and future prospects. Microbiology 158, 2695-2709.

de Haan, C.P.A., Lampen, K., Corander, J., Hänninen, M.L., 2013. Multilocus sequence types of environmental Campylobacter jejuni isolates and their similarities to those of human, poultry and bovine C. jejuni isolates. Zoonoses Public Health 60, 125-133.

de Haan, C.P.A., Kivistö, R.I., Rautelin, H., Hänninen, M., 2014. How molecular typing has changed our understanding on sources and transmission routes of campylobacteriosis in Finland. In: Sheppard, S.K. (Ed.), Campylobacter Ecology and Evolution, pp. 241-252.

EFSA, 2010. Analysis of the baseline survey on the prevalence of Campylobacter in broiler batches and of Campylobacter and Salmonella on broiler carcasses in the EU, 2008, part A: Campylobacter and Salmonella prevalence estimates. EFSA J. 8, 1503. http:// dx.doi.org/10.2903/j.efsa.2010.1503 (100 pp.).

EFSA, 2014. The European Union summary report on trends and sources of zoonoses, zoonotic agents and food-borne outbreaks in 2012. European Food Safety Authority (EFSA), Parma, Italy, European Centre for Disease Prevention and Control (ECDC), Stockholm, Sweden. EFSA J. 12, 3547. http://dx.doi.org/10.2903/j.efsa.2014.3547 (312 pp.).

EFSA Panel of Biological Hazards (BIOHAZ), 2011. Scientific opinion on Campylobacter in broiler meat production: control opinions and performance objectives and/or targets at different stages of the food chain. EFSA J. 9, 2105. http://dx.doi.org/10.2903/j.efsa. 2011.2105 (141 pp.).

Finnish Food Safety Authority Evira, 2012. Campylobacter jejuni/coli/lari- bakteerien osoittaminen siipikarjan umpisuolinäytteistä. Evira (3512/5).

Gonzalez, M., Hänninen, M., 2011. Reduction of Campylobacter jejuni counts on chicken meat treated with different seasonings. Food Control 22, 1785-1789.

Hakkinen, M., Nakari, U.M., Siitonen, A., 2009. Chickens and cattle as sources of sporadic domestically acquired Campylobacter jejuni infections in Finland. Appl. Environ. Microbiol. 75, 5244-5249.

Hermans, D., Pasmans, F., Messens, W., Martel, A., Van Immerseel, F., Rasschaert, G., Heyndrickx, M., Van Deun, K., Haesebrouck, F., 2012. Poultry as a host for the zoonotic pathogen Campylobacter jejuni. Vector Borne Zoonotic Dis. 12, 89-98.

Hörman, A., Rimhanen-Finne, R., Maunula, L., von Bonsdorff, C.H., Torvela, N., Heikinheimo, A., Hänninen, M.L., 2004. Campylobacter spp., Giardia spp., Cryptosporidium spp., noroviruses, and indicator organisms in surface water in Southwestern Finland, 2000-2001. Appl. Environ. Microbiol. 70, 87-95.

Huson, D.H., Bryant, D., 2006. Application of phylogenetic networks in evolutionary studies. Mol. Biol. Evol. 23, 254-267.

Jolley, K.A., Maiden, M.C., 2010. BIGSdb: scalable analysis of bacterial genome variation at the population level. BMC Bioinforma. 11, 595. http://dx.doi.org/10.1186/1471-210511-595.

Kärenlampi, R., Rautelin, H., Hakkinen, M., Hänninen, M.L., 2003. Temporal and geographical distribution and overlap of Penner heat-stable serotypes and pulsed-field gel electrophoresis genotypes of Campylobacter jejuni isolates collected from humans and chickens in Finland during a seasonal peak. J. Clin. Microbiol. 41, 4870-4872.
Kovanen, S.M., Kivistö, R.I., Rossi, M., Schott, T., Kärkkäinen, U.M., Tuuminen, T., Uksila, J. Rautelin, H., Hänninen, M.L., 2014. Multilocus sequence typing (MLST) and whole-genome MLST of Campylobacter jejuni isolates from human infections in three districts during a seasonal peak in Finland. J. Clin. Microbiol. 52, 4147-4154.

Levesque, S., Fournier, E., Carrier, N., Frost, E., Arbeit, R.D., Michaud, S., 2013. Campylobacteriosis in urban versus rural areas: a case-case study integrated with molecular typing to validate risk factors and to attribute sources of infection. PLoS One 8 (12), e83731. http://dx.doi.org/10.1371/journal.pone.0083731.

Llarena, A.K., Huneau, A., Hakkinen, M., Hänninen, M.L., 2015. Predominant Campylobacter jejuni sequence types persist in Finnish chicken production. PLoS One 10 (2) e0116585. http://dx.doi.org/10.1371/journal.pone.0116585.

Maa- ja Metsätalousministeriö, 2007. Maa ja Metsätalousministeriön asetus broilereiden kampylobakteerivalvonnasta. 10/EEO/2007.

Mughini Gras, L., Smid, J.H., Wagenaar, J.A., de Boer, A.G., Havelaar, A.H., Friesema, I.H., French, N.P., Busani, L., van Pelt, W., 2012. Risk factors for campylobacteriosis of chicken, ruminant, and environmental origin: a combined case-control and source attribution analysis. PLoS One 7 (8), e42599. http://dx.doi.org/10.1371/journal pone.0042599.

Revez, J., Schott, T., Llarena, A.K., Rossi, M., Hänninen, M.L., 2013. Genetic heterogeneity of Campylobacter jejuni NCTC 11168 upon human infection. Infect. Genet. Evol. 16, 305-309.

Revez, J., Zhang, J., Schott, T., Kivistö, R., Rossi, M., Hänninen, M.L., 2014. Genomic variation between milkborne outbreak-associated Campylobacter jejuni isolates. J. Clin. Microbiol. 52, 2782-2786.

Schönberg-Norio, D., Takkinen, J., Hänninen, M.L., Katila, M.L., Kaukoranta, S.S., Mattila, L., Rautelin, H., 2004. Swimming and Campylobacter infections. Emerg. Infect. Dis. 10, 1474-1477.

Sheppard, S.K., Dallas, J.F., MacRae, M., McCarthy, N.D., Sproston, E.L., Gormley, F.J., Strachan, N.J.C., Ogden, I.D., Maiden, M.C.J., Forbes, K.J., 2009. Campylobacter genotypes from food animals, environmental sources and clinical disease in Scotland 2005/6. Int. J. Food Microbiol. 134, 96-103.

Sheppard, S.K., Jolley, K.A., Maiden, M.C.J., 2012. A gene-by-gene approach to bacterial population genomics: whole genome MLST of Campylobacter. Gene 3, 261-277.

Skirrow, M.B., 1977. Campylobacter enteritis: a "new" disease. Br. Med. J. 2, 9-11.

Strachan, N.J., Rotariu, O., Smith-Palmer, A., Cowden, J., Sheppard, S.K., O'Brien, S.J., Maiden, M.C., Macrae, M., Bessell, P.R., Matthews, L., Reid, S.W., Innocent, G.T. Ogden, I.D., Forbes, K.J., 2013. Identifying the seasonal origins of human campylobacteriosis. Epidemiol. Infect. 141, 1267-1275.

Vierikko, A., Hänninen, M.L., Siitonen, A., Ruutu, P., Rautelin, H., 2004. Domestically acquired Campylobacter infections in Finland. Emerg. Infect. Dis. 10, 127-130.

Wilson, D.J., Gabriel, E., Leatherbarrow, A.J.H., Cheesbrough, J., Gee, S., Bolton, E., Fox, A., Fearnhead, P., Hart, C.A., Diggle, P.J., 2008. Tracing the source of campylobacteriosis PLoS Genet. 4 (9), e1000203. http://dx.doi.org/10.1371/journal.pgen.1000203.

Zhang, J., Halkilahti, J., Hänninen, M.L., Rossi, M., 2015. Refinement of whole-genome multilocus sequence typing analysis by addressing gene paralogy. J. Clin. Microbiol. $53,1765-1767$. 